PROCEEDINGS OF THE

AMERICAN MATHEMATICAL SOCIETY

Volume 125, Number 2, February 1997, Pages 397-406

S 0002-9939(97)03947-6

\title{
GENERALIZED UPPER AND LOWER SOLUTION METHOD FOR THE FORCED DUFFING EQUATION
}

\author{
CHENGWEN WANG
}

(Communicated by Hal L. Smith)

\begin{abstract}
This paper gives the generalized upper and lower solution method for the forced Duffing equation$$
x^{\prime \prime}+k x^{\prime}+f(t, x)=0,
$$

and obtains existence theorems for $T$-periodic solutions, where $f$ is a Carathéodory function. Our results generalize or extend some famous results obtained by Mawhin(1985), Habets(1990), Nkashama(1989) and Nieto(1990).
\end{abstract}

\section{INTRODUCTION}

In this paper, we propose a generalized upper and lower solution method for the existence of periodic solutions of the Duffing equation

$$
\begin{gathered}
x^{\prime \prime}+k x^{\prime}+f(t, x)=0 \quad \text { a.e. on } \quad I=[0, T], \\
x(0)=x(T), \quad x^{\prime}(0)=x^{\prime}(T)
\end{gathered}
$$

where $f:[0, T] \times \mathbb{R} \rightarrow \mathbb{R}$ is a Carathéodory function and $k \in \mathbb{R} \backslash\{0\}$.

We recall (see [6]) that $f:[0, T] \times \mathbb{R} \rightarrow \mathbb{R}$ is called a Carathéodory function if $f(\cdot, x)$ is measurable for all $x \in \mathbb{R}$ and $f(t, \cdot)$ is continuous for a.e. $t \in[0, T]$.

Mawhin in [5] first gave the upper and lower solution method for (1.1)-(1.2) under the continuous case. Nkashama generalized this method to the Carathéodory case in [6] for the first order differential equation. In [3], Habets et al. obtained similar results to the Carathéodory case for the Liénard equation, which is more general than the Duffing equation. But their results are only applicable to the case $k>0$. In [7], Nieto et al. extended these results in a way.

In this paper, we propose a generalized upper and lower solution method for (1.1)-(1.2) under a Carathéodory condition for $k \in \mathbb{R} \backslash\{0\}$. The upper and lower solutions may no longer be periodic and the above mentioned results are generalized. In addition, we give an applicable example in the last section.

Finally, let us give the following notation for convenience. Let $I=[0, T] . \mathbb{R}$ denotes all real numbers. $L^{p}(I), p=1,2$, denotes the usual Lebesgue space with norm $|\cdot|_{L^{p}}=\left(\int_{0}^{T}|x(t)|^{p} d t\right)^{\frac{1}{p}}$. The Sobolev spaces $W^{2, i}(I)(i=1,2)$ are defined

Received by the editors November 16, 1994.

1991 Mathematics Subject Classification. Primary 34B15, 34C25.

(C) 1997 American Mathematical Society 
by

$$
W^{2, i}(I)=\left\{x \mid x \in L^{i}(I), x^{(j)} \in L^{i}(I), j=1,2\right\}
$$

with norm

$$
|x|_{W^{2, i}}=\left(\sum_{j=0}^{2}\left|x^{(j)}\right|_{L^{i}}^{i}\right)^{\frac{1}{i}},
$$

where $x^{(j)}$ denotes the distributional derivatives of $x$. Let $C(I)$ denote real valued continuous functions on $I$, and let

$$
|x|_{\infty}=\max \{|x(t)| \mid t \in I\} .
$$

\section{Definitions And Theorems}

In this section, we give the definitions of generalized upper and lower solutions and state our main results.

First, we suppose that $f(t, x)$ is a Carathéodory function satisfying the growth restriction, i.e., for each real constant $r \geq 0$, there exists a function $h_{r} \in L^{1}(I)$ such that for a.e. $t \in I$ and all $x \in \mathbb{R}$ with $|x| \leq r$, we have

$$
|f(t, x)| \leq h_{r}(t) .
$$

We call a function $x: I \rightarrow \mathbb{R}$ the solution of (1.1)-(1.2) if it is a continuously differentiable function such that $x^{\prime}$ is absolutely continuous and (1.1)-(1.2) hold.

Definition 2.1. Let $a: I \rightarrow \mathbb{R}, b: I \rightarrow \mathbb{R}$ be functions of class $C^{1}$ with absolutely continuous derivatives such that for all $t \in I$

$$
a(t) \leq b(t) .
$$

Such functions $a$ and $b$ are called lower and upper solutions respectively, if they satisfy

$$
\begin{aligned}
& a^{\prime \prime}(t)+k a^{\prime}(t)+f(t, a(t)) \geq 0 \quad \text { a.e. } \quad t \in I, \\
& a(0)=a(T), \quad a^{\prime}(0) \geq a^{\prime}(T),
\end{aligned}
$$

and

$$
\begin{aligned}
& b^{\prime \prime}(t)+k b^{\prime}(t)+f(t, b(t)) \leq 0 \quad \text { a.e. } \quad t \in I, \\
& b(0)=b(T), \quad b^{\prime}(0) \leq b^{\prime}(T) .
\end{aligned}
$$

Theorem 2.1. Assume that there exist a lower solution $a(t)$ and an upper solution $b(t)$ defined by Definition 2.1 for (1.1)-(1.2). Then the periodic BVP (1.1)-(1.2) has at least one solution $x(t)$ such that $a(t) \leq x(t) \leq b(t)$ for all $t \in I$.

Remark. Although Theorem 2.1 can be a special case of more general results obtained in [7] and the references therein, we will give it a full proof in our way so as to prove our main result (Theorem 2.2).

Definition 2.2. Let $a_{1}(t), b_{1}(t) \in C^{1}$ with absolutely continuous derivatives and $a_{1}(t) \leq b_{1}(t)$ for all $t \in I$. Such $a_{1}(t)$ and $b_{1}(t)$ are called reversedly lower and upper solutions respectively, if they satisfy

$$
\begin{aligned}
& a_{1}^{\prime \prime}(t)+k a_{1}^{\prime}(t)+f\left(t, a_{1}(t)\right) \leq 0 \quad \text { for } \quad \text { a.e. } \quad t \in I, \\
& a_{1}(0)=a_{1}(T), \quad a_{1}^{\prime}(0) \leq a_{1}^{\prime}(T)
\end{aligned}
$$


and

$$
\begin{aligned}
& b_{1}^{\prime \prime}(t)+k b_{1}^{\prime}(t)+f\left(t, b_{1}(t)\right) \geq 0 \quad \text { for } \quad \text { a.e. } t \in I, \\
& b_{1}(0)=b_{1}(T), \quad b_{1}^{\prime}(0) \geq b_{1}^{\prime}(T) .
\end{aligned}
$$

Theorem 2.2. Assume that $f(t, x)$ has the nonincreasing property with respect to $x$. If there exist a reversedly lower solution $a_{1}(t)$ and a reversedly upper solution $b_{1}(t)$ for (1.1)-(1.2) defined by Definition 2.2, then the periodic BVP (1.1)-(1.2) has at least one solution $x(t)$ such that $a_{1}(t) \leq x(t) \leq b_{1}(t)$ for all $t \in I$.

\section{Proof of the Theorems}

Proof of Theorem 2.1. First, let us define the function $c: \mathbb{R}^{3} \rightarrow \mathbb{R}$ by

$$
c(r, x, R)= \begin{cases}R & \text { if } x>R \\ x & \text { if } r \leq x \leq R, \\ r & \text { if } x<r\end{cases}
$$

and define

$$
F(t, x)=f(t, c(a(t), x, b(t))) .
$$

It is evident that $F(t, x)$ is also a Carathéodory function.

Now, we modify the periodic boundary value problem (1.1)-(1.2) to the problem

$$
\begin{gathered}
x^{\prime \prime}+k x^{\prime}+F(t, x)=x-c(a(t), x, b(t)) \quad \text { a.e. } \quad t \in I, \\
x(0)=x(T), \quad x(0)=c\left(a(0), x(0)+x^{\prime}(0)-x^{\prime}(T), b(0)\right) .
\end{gathered}
$$

We can prove that (3.3)-(3.4) is equivalent to (1.1)-(1.2) for $t \in I$ and $a(t) \leq$ $x \leq b(t)$. It is sufficient to show that any solution $x(t)$ of (3.3)-(3.4) satisfies $a(t) \leq x(t) \leq b(t)$ for all $t \in I$, and (1.2).

In fact, it is clear that, from (3.4) and (3.1), $a(0) \leq x(0) \leq b(0)$ and, from (3.4),

$$
a(T)=a(0) \leq x(0)=x(T) \leq b(0)=b(T) .
$$

In order to show $a(t) \leq x(t) \leq b(t)$ for $t \in(0, T)$, let $y: I \rightarrow \mathbb{R}$ be $y(t)=$ $\exp \left(\frac{k}{2} t\right) x(t)$. Putting $C=\exp \left(\frac{k}{2} T\right)$, the modified BVP (3.3)-(3.4) can then be changed into

$$
\begin{gathered}
y^{\prime \prime}-\frac{k^{2}}{4} y+F\left(t, y \exp \left(-\frac{k}{2} t\right)\right) \exp \left(\frac{k}{2} t\right) \\
=y-c\left(a(t), y(t) \exp \left(-\frac{k}{2} t\right), b(t)\right) \exp \left(\frac{k}{2} t\right) \quad \text { a.e. } \quad t \in I, \\
y(T)=C y(0), \quad y(0)=c\left(\alpha(0), y(0)+y^{\prime}(0)-\frac{1}{C} y^{\prime}(T), \beta(0)\right) .
\end{gathered}
$$

Letting $\alpha(t)=\exp \left(\frac{k}{2} t\right) a(t)$ and $\beta(t)=\exp \left(\frac{k}{2} t\right) b(t)$, then $\alpha(t)$ and $\beta(t)$ are lower and upper solutions of BVP (3.6)-(3.7) respectively which satisfy the following relations:

$$
\begin{aligned}
& \alpha^{\prime \prime}-\frac{k^{2}}{4} \alpha+F\left(t, \alpha \exp \left(-\frac{k}{2} t\right)\right) \exp \left(\frac{k}{2} t\right) \geq 0 \quad \text { a.e. } t \in I, \\
& \alpha(T)=C \alpha(0), \quad \alpha(0) \geq c\left(\alpha(0), \alpha(0)+\alpha^{\prime}(0)-\frac{1}{C} \alpha^{\prime}(T), \beta(0)\right),
\end{aligned}
$$


and

$$
\begin{gathered}
\beta^{\prime \prime}-\frac{k^{2}}{4} \beta+F\left(t, \beta \exp \left(-\frac{k}{2} t\right)\right) \exp \left(\frac{k}{2} t\right) \leq 0 \quad \text { a.e. } t \in I, \\
\beta(T)=C \beta(0), \quad \beta(0) \leq c\left(\alpha(0), \beta(0)+\beta^{\prime}(0)-\frac{1}{C} \beta^{\prime}(T), \beta(0)\right),
\end{gathered}
$$

and $\alpha(t) \leq \beta(t) \quad$ for $\quad t \in I$.

It follows from (3.5) that $\alpha(0) \leq y(0) \leq \beta(0)$ and $\alpha(T) \leq y(T) \leq \beta(T)$. Suppose that there exists some $t_{0} \in I$ such that $y\left(t_{0}\right)>\beta\left(t_{0}\right)$. Then, by continuity, there exist $t_{1}$ and $t_{2} \in I, t_{1}<t_{0}<t_{2}$, such that $y(t)-\beta(t)>0$ for all $t \in\left(t_{1}, t_{2}\right)$, and $y\left(t_{1}\right)-\beta\left(t_{1}\right)=y\left(t_{2}\right)-\beta\left(t_{2}\right)=0$. Thus there exists a subset $I_{0}$ in $\left(t_{1}, t_{2}\right)$ with positive measure such that for all $t \in I_{0}$,

$$
y^{\prime \prime}(t)-\beta^{\prime \prime}(t)<0 .
$$

But, by (3.9), for a.e. $t \in I$ such that $y(t)>\beta(t)$ we have

$$
\begin{aligned}
y^{\prime \prime}(t) & =\frac{k^{2}}{4} y(t)-F\left(t, y(t) \exp \left(-\frac{k}{2} t\right)\right) \exp \left(\frac{k}{2} t\right)+y(t)-c(\alpha(t), y(t), \beta(t)) \\
& >\frac{k^{2}}{4} \beta(t)-F\left(t, \beta(t) \exp \left(-\frac{k}{2} t\right)\right) \exp \left(\frac{k}{2} t\right) \\
& \geq \beta^{\prime \prime}(t),
\end{aligned}
$$

i.e.

$$
y^{\prime \prime}(t)-\beta^{\prime \prime}(t)>0
$$

for a.e. $t \in\left(t_{1}, t_{2}\right)$, which conflicts with (3.10). Therefore, $y(t) \leq \beta(t)$ for all $t \in I$.

A similar proof shows that $y(t) \geq \alpha(t)$ for all $t \in I$.

Now, we show that $x^{\prime}(0)=x^{\prime}(T)$. According to what we have proved, i.e., $a(t) \leq x(t) \leq b(t)$ for $t \in[0, T]$, and the definition of $c(r, x, R)$, what we have to prove is to exclude the case $x(0)=b(0), x^{\prime}(0)>x^{\prime}(T)$ or $x(0)=a(0), x^{\prime}(0)<x^{\prime}(T)$.

In fact, $x(0)=b(0)$ implies that $x^{\prime}(0) \leq b^{\prime}(0)$. If not, then there exists a $t_{0}^{\prime}>0$ such that $x(t)>b(t)$ for $t \in\left(0, t_{0}^{\prime}\right)$, a contradiction. Similarly, $x(T)=b(T)$ implies that $x^{\prime}(T) \geq b^{\prime}(T)$. Therefore, $x^{\prime}(0) \leq b^{\prime}(0) \leq b^{\prime}(T) \leq x^{\prime}(T)$.

Also, we can prove that $x^{\prime}(0) \geq a^{\prime}(0) \geq a^{\prime}(T) \geq x^{\prime}(T)$ if $x(0)=a(0)$.

So, (3.3)-(3.4) is equivalent to (1.1)-(1.2).

Next, we prove that the modified periodic BVP (3.3)-(3.4) has at least one solution by applying Leray-Schauder degree theory. Basing on this consideration, we discuss the homotopy

$$
\begin{aligned}
x^{\prime \prime}(t)+k x^{\prime}(t) & =(1-\lambda) x(t)+\lambda[x(t)-F(t, x(t))-c(a(t), x(t), b(t))], \\
& x(0)-x(T)=x^{\prime}(0)-x^{\prime}(T)=0
\end{aligned}
$$

which is equivalent to

$$
\begin{gathered}
x^{\prime \prime}(t)+k x^{\prime}(t)-x(t)=-\lambda[F(t, x(t))+c(a(t), x(t), b(t))], \\
x(0)-x(T)=x^{\prime}(0)-x^{\prime}(T)=0
\end{gathered}
$$

where $\lambda \in[0,1]$.

Let $x(t)$ be a solution of problem (3.12)-(3.13) for $\lambda=1$. We have to prove that

$$
a(0) \leq x(0) \leq b(0),
$$

which guarantees that $x(t)$ satisfies (3.4). 
In fact, if $x(t)<a(t)$ for all $t \in I$, then Eq. $(3.12)_{\lambda=1}$ can be simplified to

$$
x^{\prime \prime}(t)+k x^{\prime}(t)=(x(t)-a(t))-f(t, a(t))
$$

for a.e. $t \in I$. By the first formula in (2.3), we have $x^{\prime \prime}(t)+k x^{\prime}(t) \leq(x(t)-a(t))+$ $a^{\prime \prime}(t)+k a^{\prime}(t)<a^{\prime \prime}(t)+k a^{\prime}(t)$, i.e.,

$$
x^{\prime \prime}(t)+k x^{\prime}(t)<a^{\prime \prime}(t)+k a^{\prime}(t)
$$

for a.e. $t \in I$.

Integrating (3.16) from 0 to $T$, we have $a^{\prime}(0)<a^{\prime}(T)$, which conflicts with the second formula in (2.3). Therefore, if $x(0)<a(0)$, there must exist a $t_{01} \in I$ such that $x\left(t_{01}\right)=a\left(t_{01}\right)$, and $x(t)<a(t)$ for $0 \leq t<t_{01}$. Similarly, it follows from the fact $x(0)=x(T), a(0)=a(T)$ that there exists a $t_{02} \in I, t_{01}<t_{02}<T$, such that $x\left(t_{02}\right)=a\left(t_{02}\right)$, and $x(t)<a(t)$ for $t_{02}<t \leq T$. The same argument shows that (3.16) holds for a.e. $t \in\left[0, t_{01}\right) \cup\left(t_{02}, T\right]$.

Noticing that $x(t)-a(t)$ increases from negative to nonnegative as $t \rightarrow t_{01}^{-}$, we can conclude that $x^{\prime}\left(t_{01}\right)-a^{\prime}\left(t_{01}\right) \geq 0$. Therefore, if $x^{\prime}(0)-a^{\prime}(0)<0$, then there exists a $\bar{t}_{01} \in\left(0, t_{01}\right)$ such that $x\left(\bar{t}_{01}\right)-a\left(\bar{t}_{01}\right)$ is a minimum which is smaller than $x(0)-a(0)$. Similarly, we can conclude that $x^{\prime}\left(t_{02}\right)-a^{\prime}\left(t_{02}\right) \leq 0$ because $x(t)-a(t)$ decreases from nonnegative to negative as $t$ increases from $t_{02}$ to $t_{02}^{+}$. Therefore, if $x^{\prime}(0)-a^{\prime}(0)>0$, then $x^{\prime}(T)-a^{\prime}(T) \geq x^{\prime}(0)-a^{\prime}(0)>0$, which implies there exists a $\bar{t}_{02} \in\left(t_{02}, T\right)$ such that $x\left(\bar{t}_{02}\right)-a\left(\bar{t}_{02}\right)$ is a minimum not greater than $x(T)-a(T)$. Finally, supposing $x^{\prime}(0)-a^{\prime}(0)=0$, then $x^{\prime}(T)-a^{\prime}(T) \geq 0$ by $(2.3)^{\prime}$. Therefore, whether $x(0)-a(0)$ is a minimum or not, there exists a minimum point $\bar{t}_{01} \in[0, T]$ for $x(t)-a(t)$.

Now, given a minimum point $\widetilde{t}_{0}$ as above, for any $\xi$ sufficiently close to and smaller than $\widetilde{t}_{0}$, which implies $x^{\prime}(\xi)-a^{\prime}(\xi) \leq 0$, there exists a $\zeta$ sufficiently close to and greater than $\widetilde{t}_{0}$, which implies $x^{\prime}(\zeta)-a^{\prime}(\zeta) \geq 0$, such that $x(\xi)-a(\xi)=$ $x(\zeta)-a(\zeta)$. (In case $\widetilde{t}_{0}=T$, we may take a $\zeta$ which is sufficiently close to and greater than 0.) Integrating (3.16) from $\xi$ to $\zeta$ (in case $\widetilde{t}_{0}=T$, from $\xi$ to $\zeta+T$ ), we obtain $\left(x^{\prime}(\zeta)-a^{\prime}(\zeta)\right)-\left(x^{\prime}(\xi)-a^{\prime}(\xi)\right) \leq 0$, which conflicts with the choice of $\xi$ and $\zeta$.

Therefore, $x(0) \geq a(0)$; similarly, $x(0) \leq b(0)$. Hence, Eq. $(3.12)_{\lambda=1}-(3.13)$ is equivalent to Eq. (3.3)-(3.4), and also to Eq. (1.1)-(1.2).

Let us first, as in [2], define the differential operator $L: W_{1}^{2,1}(I) \subset L^{2}(I) \rightarrow L^{1}(I)$ by

$$
L x=x^{\prime \prime}+k x^{\prime}-x
$$

where $W_{1}^{2,1}(I) \subset W^{2,1}(I)$ is the Sobolev function space of period- $T$ defined by $W_{1}^{2,1}(I)=\left\{x \in W^{2,1}(I) \mid x^{\prime}(0)-x^{\prime}(T)=x(0)-x(T)=0\right\}$. It is clear that $L$ is a Fredholm operator of index 0 . The spectrum of $L$ is defined by $\sigma(L)$. Obviously, $K=L^{-1}: L^{1}(I) \rightarrow W_{1}^{2,1}(I)$ exists and is continuous because $0 \notin \sigma(L)$.

Now define $N: L^{2}(I) \rightarrow L^{1}(I)$ by $N x=F(\cdot, x(\cdot))+c(a(\cdot), x(\cdot), b(\cdot))$. Then (3.12)-(3.13) can be written in the equivalent form

$$
L x=-\lambda N x
$$

where $\lambda \in[0,1]$ and $x \in W_{1}^{2,1}(I)$. It follows from the discussion above that $K$ : $L^{1}(I) \rightarrow L^{2}(I)$ is compact since $W_{1}^{2,1}(I)$ is compactly embedded into $L^{2}(I)$ and 
(3.18) is equivalent to

$$
x=-\lambda K N x .
$$

We shall prove that all the solutions of Eq. (3.19) are bounded independently of $\lambda \in[0,1]$, i.e., to find a priori bounds for solutions of (3.19).

Let $x \in W_{1}^{2,1}(I)$ be a solution of $(3.12)-(3.13)$ for some $\lambda \in[0,1]$. It follows from $a(t) \leq c(a(t), x(t), b(t)) \leq b(t)$ for all $t \in I$ with the continuity of $a$ and $b$, the definition of $F$ and relation (2.1), that

$$
|-\lambda[F(\cdot, x(\cdot))+c(a(\cdot), x(\cdot), b(\cdot))]|_{L^{2}} \leq|d|_{L^{2}}
$$

for some $d \in L^{2}(I)$ which depends only on $a$ and $b$ but not on $\lambda$ or $x$.

Also, because of the existence of $K=L^{-1}$, taking $r=1 /\|K\|$, we have

$$
\left|x^{\prime \prime}+k x^{\prime}-x\right|_{L^{2}} \geq r|x|_{W^{2,1}} .
$$

Therefore, it follows from (3.20) and (3.21) that

$$
|x|_{W^{2,1}} \leq r^{-1}|d|_{L^{2}}
$$

which proves the a priori boundedness of the solutions of (3.12)-(3.13).

Let $\Omega=\left\{\left.x \in W^{2,2}(I)|| x\right|_{W^{2,1}}<r^{-1}|d|_{L^{2}(I)}\right\}$. We have proved that

$$
d\left(I+L^{-1} N, \Omega, 0\right)=d(I, \Omega, 0)=1
$$

because of the homotopy invariance of the Leray-Schauder degree, where $d(\cdot, \cdot, \cdot)$ denotes the Leray-Schauder degree. By the generalized Leray-Schauder continuation theorem given by Mawhin [1], we obtain the existence of the solution of (1.1)-(1.2). The proof is complete.

In order to prove Theorem 2.2, we first have

Lemma. Assume that $f(t, x)$ has the nonincreasing property with respect to $x$. Let $a_{1}(t)$ and $b_{1}(t)$ be reversedly lower and upper solutions for (1.1)-(1.2) respectively defined by Definition 2.2, and let $\alpha_{1}(t)=\exp \left(\frac{k}{2} t\right) a_{1}(t), \beta_{1}(t)=\exp \left(\frac{k}{2} t\right) b_{1}(t)$, and $y(t)=\exp \left(\frac{k}{2} t\right) x(t)$, where $x(t)$ is any solution of $(3.3)-(3.4)$ and $a(t)$ and $b(t)$ in (3.3) are now (and in the following) replaced by $a_{1}(t)$ and $b_{1}(t)$. Then

$$
y^{\prime \prime}(t)-\alpha_{1}^{\prime \prime}(t)>0
$$

holds for almost all $t \in I$ such that $y(t)-\alpha_{1}(t)>0$; and

$$
y^{\prime \prime}(t)-\beta_{1}^{\prime \prime}(t)<0
$$

holds for almost all $t \in I$ such that $y(t)-\beta_{1}(t)<0$.

Proof. In fact, let $C=\exp \left(\frac{k}{2} T\right)$, such $\alpha_{1}(t)$ and $\beta_{1}(t)$ satisfy

$$
\begin{gathered}
\alpha_{1}^{\prime \prime}-\frac{k^{2}}{4} \alpha_{1}+F\left(t, \alpha_{1} \exp \left(-\frac{k}{2} t\right)\right) \exp \left(\frac{k}{2} t\right) \leq 0 \\
\alpha_{1}(T)=C \alpha_{1}(0), \quad \alpha_{1}(0) \leq c\left(\alpha_{1}(0), \alpha_{1}(0)+\alpha_{1}^{\prime}(0)-\frac{1}{C} \alpha_{1}^{\prime}(T), \beta_{1}(0)\right),
\end{gathered}
$$


and

$(3.9)^{\prime}$

$$
\begin{gathered}
\beta_{1}^{\prime \prime}-\frac{k^{2}}{4} \beta_{1}+F\left(t, \beta_{1} \exp \left(-\frac{k}{2} t\right)\right) \exp \left(\frac{k}{2} t\right) \geq 0, \\
\beta_{1}(T)=C \beta_{1}(0), \quad \beta_{1}(0) \geq c\left(\alpha_{1}(0), \beta_{1}(0)+\beta_{1}^{\prime}(0)-\frac{1}{C} \beta_{1}^{\prime}(T), \beta_{1}(0)\right),
\end{gathered}
$$

and

$$
\alpha_{1}(t) \leq \beta_{1}(t) \quad \text { for } \quad t \in I \text {. }
$$

Let $\triangle=\left\{t \in I \mid y(t)<\beta_{1}(t)\right\}$. Then according to the nonincreasing property of $f$ with respect to the second variable, we have

$$
\begin{gathered}
y^{\prime \prime}(t)=\frac{k^{2}}{4} y(t)-F\left(t, y(t) \exp \left(-\frac{k}{2} t\right)\right) \exp \left(\frac{k}{2} t\right)+y(t)-c\left(\alpha_{1}(t), y(t), \beta_{1}(t)\right) \\
<\frac{k^{2}}{4} \beta_{1}(t)-F\left(t, \beta_{1}(t) \exp \left(-\frac{k}{2} t\right)\right) \exp \left(\frac{k}{2} t\right) \leq \beta_{1}^{\prime \prime}(t)
\end{gathered}
$$

for a.e. $t \in \triangle$, which proves inequality (3.23).

Similarly, we can prove inequality (3.22). The proof of the Lemma is complete.

Proof of Theorem 2.2. Let $y(t)=\exp \left(\frac{k}{2} t\right) x(t)$, where $x(t)$ is any solution of (3.3)(3.4). Similarly to the proof of Theorem 2.1, we first show that $\alpha_{1}(t) \leq y(t) \leq \beta_{1}(t)$ for all $t \in I$, where $\alpha_{1}(t)$ and $\beta_{1}(t)$ satisfy the inequalities $(3.8)^{\prime}$ and $(3.9)^{\prime}$.

Firstly, we also have $\alpha_{1}(0) \leq y(0) \leq \beta_{1}(0), \quad \alpha_{1}(T) \leq y(T) \leq \beta_{1}(T)$.

Now suppose that $y^{\prime}(0)-\alpha_{1}^{\prime}(0)>0$. Then $y(t)-\alpha_{1}(t)>0$ for all $t \in(0, T]$. In fact, if there exists $\widetilde{t}_{0} \in(0, T]$ such that $y\left(\widetilde{t}_{0}\right)-\alpha_{1}\left(\widetilde{t}_{0}\right)=y(0)-\alpha_{1}(0)$ and $y(t)-\alpha_{1}(t)>y(0)-\alpha_{1}(0)$ for $t \in\left(0, \widetilde{t}_{0}\right)$, then by Rolle's Theorem there exists $\bar{t}_{0}, 0<\bar{t}_{0}<\widetilde{t}_{0}$, such that

$$
y^{\prime}\left(\bar{t}_{0}\right)-\alpha_{1}^{\prime}\left(\bar{t}_{0}\right)=0 .
$$

But $y(t)-\alpha_{1}(t)>y(0)-\alpha_{1}(0) \geq 0$ for $t \in\left(0, \bar{t}_{0}\right]$, which implies by our Lemma that $y_{1}^{\prime \prime}(t)-\alpha_{1}^{\prime \prime}(t)>0$ for $t \in\left(0, \bar{t}_{0}\right]$ and therefore $y^{\prime}\left(\bar{t}_{0}\right)-\alpha_{1}^{\prime}\left(\bar{t}_{0}\right)>y^{\prime}(0)-\alpha_{1}^{\prime}(0)>0$, a contradiction to (3.24). Hence, $y(t)-\alpha_{1}(t)>y(0)-\alpha_{1}(0) \geq 0$ for all $t \in I$. Thus the proof will be completed.

Now, suppose that $y^{\prime}(0)-\alpha_{1}^{\prime}(0) \leq 0$, and there exist $\widetilde{t}_{1}, \widetilde{t}_{2}$ and $\widetilde{t}_{3} \in I, \widetilde{t}_{1}<\widetilde{t}_{2}<\widetilde{t}_{3}$, such that $y\left(\widetilde{t}_{1}\right)-\alpha_{1}\left(\widetilde{t}_{1}\right)=y\left(\widetilde{t}_{3}\right)-\alpha_{1}\left(\widetilde{t}_{3}\right)=0, y\left(\widetilde{t}_{2}\right)-\alpha_{1}\left(\widetilde{t}_{2}\right)<0$ and $y(t)-\alpha_{1}(t)>0$ for $t \in\left(0, \widetilde{t}_{1}\right) \cup\left(\widetilde{t}_{3}, T\right)$. Because of $y(T)-\alpha_{1}(T)=C\left(y(0)-\alpha_{1}(0)\right)$, it follows that $\widetilde{t}_{1}=0$ if and only if $\widetilde{t}_{3}=T$.

If $\widetilde{t}_{1} \neq 0$ and $\widetilde{t}_{3} \neq T$, then $y^{\prime}\left(\widetilde{t}_{3}\right)-\alpha_{1}^{\prime}\left(\widetilde{t}_{3}\right) \geq 0$ because $y(t)-\alpha_{1}(t)>0$ for $\widetilde{t}_{3}<t<T$ and $y\left(\widetilde{t}_{3}\right)-\alpha_{1}\left(\widetilde{t}_{3}\right)=0$. And $y(t)-\alpha_{1}(t)>0$ for $\widetilde{t}_{3}<t \leq T$ implies $y^{\prime \prime}(t)-\alpha_{1}^{\prime \prime}(t)>0$ for $\widetilde{t}_{3}<t \leq T$ by our Lemma, and therefore $y^{\prime}(T)-\alpha_{1}^{\prime}(T)>$ $y^{\prime}\left(\widetilde{t_{3}}\right)-\alpha_{1}^{\prime}\left(\widetilde{t_{3}}\right) \geq 0$. It follows from $(3.8)^{\prime}$ that $\alpha_{1}^{\prime}(T) \geq C \alpha_{1}^{\prime}(0)$ which implies

$$
0<y^{\prime}(T)-\alpha_{1}^{\prime}(T) \leq C\left(y^{\prime}(0)-\alpha_{1}^{\prime}(0)\right)
$$

which contradicts our assumption $y^{\prime}(0)-\alpha_{1}^{\prime}(0) \leq 0$ because $C>0$.

If $\widetilde{t}_{1}=0, \widetilde{t}_{3}=T$ and $y^{\prime}(0)-\alpha_{1}^{\prime}(0)<0$, then $y^{\prime}(T)-\alpha_{1}^{\prime}(T) \geq 0$. In fact, if $y^{\prime}(T)-\alpha_{1}^{\prime}(T)<0$, then there exists a $\widetilde{t}_{4}, 0<\widetilde{t}_{4}<T$, such that $y\left(\widetilde{t}_{4}\right)-\alpha_{1}\left(\widetilde{t}_{4}\right)=$ $0, y^{\prime}\left(\widetilde{t}_{4}\right)-\alpha_{1}^{\prime}\left(\widetilde{t}_{4}\right) \geq 0$ and $y(t)-\alpha_{1}(t)>0$ for $t \in\left(\widetilde{t}_{4}, T\right)$ because of $y(T)-\alpha_{1}(T)=$ $y(0)-\alpha_{1}(0)=0$. Again, by our Lemma, we have $y^{\prime \prime}(t)-\alpha_{1}^{\prime \prime}(t)>0$ for all $t \in\left(\widetilde{t}_{4}, T\right)$, 
which implies $y^{\prime}\left(\widetilde{t}_{4}\right)-\alpha_{1}^{\prime}\left(\widetilde{t}_{4}\right)<0$, a contradiction. Therefore, $y^{\prime}(T)-\alpha_{1}^{\prime}(T) \geq 0$, and again by $(3.25)$ we have a contradiction.

Finally, if $\widetilde{t}_{1}=0, \widetilde{t}_{3}=T$ and $y^{\prime}(0)-\alpha_{1}^{\prime}(0)=0$, then $y^{\prime}(0)=\alpha_{1}^{\prime}(0), y(0)=\alpha_{1}(0)$. By (3.6), (3.8)', the nonincreasing property of $f$ and differential inequalities (see, for example, Corollary 4.3 [4, Chapter III] and the exercises following it), we get $y(t) \geq \alpha_{1}(t)$ for all $t \in I$.

Therefore, we prove that $y(t)-\alpha_{1}(t) \geq 0$ for all $t \in I$, and, similarly, $y(t)-\beta_{1}(t) \leq$ 0 for all $t \in I$.

Now, let $x(t)$ be a solution of problem (3.12)-(3.13) for $\lambda=1$. Similarly to the proof in Theorem 2.1, we shall show that

$$
a_{1}(0) \leq x(0) \leq b_{1}(0) .
$$

In fact, if $x(t)<a_{1}(t)$ for all $t \in I$, then $x(t)<b_{1}(t)$ for all $t \in I$ according to Definition 2.2. Therefore, Eq. (3.12) $)_{\lambda=1}$ can also be simplified into

$$
x^{\prime \prime}(t)+k x^{\prime}(t)=\left(x(t)-a_{1}(t)\right)-f\left(t, a_{1}(t)\right)
$$

for a.e. $t \in I$. By the nonincreasing property of $f(t, x)$ and the first formulae in $(2.3)^{\prime}$ and $(2.4)^{\prime}$, we obtain

$$
x^{\prime \prime}(t)+k x^{\prime}(t)<-f\left(t, a_{1}(t)\right) \leq-f\left(t, b_{1}(t)\right) \leq b_{1}^{\prime \prime}(t)+k b_{1}^{\prime}(t),
$$

i.e.

$$
x^{\prime \prime}(t)+k x^{\prime}(t)<b_{1}^{\prime \prime}(t)+k b_{1}^{\prime}(t) .
$$

Integrating $(3.16)^{\prime}$ from 0 to $T$, we have $b_{1}^{\prime}(0)<b_{1}^{\prime}(T)$, which conflicts with the second formula in $(2.4)^{\prime}$. Similarly to the proof following (3.16), we can prove that $x(0)<a_{1}(0)$ or $x(0)>b_{1}(0)$ is impossible. Therefore, $a_{1}(0) \leq x(0) \leq b_{1}(0)$, which guarantees the equivalence of $(3.12)_{\lambda=1}-(3.13)$ and (3.3)-(3.4).

The proof of Theorem 2.2 is complete.

\section{Applicable example}

In this section, we study the existence of solutions to the following periodic boundary value problem for the second order Duffing equation

$$
\begin{gathered}
x^{\prime \prime}+k x^{\prime}+g(t, x)=s \quad \text { a.e. on }[0, T], \\
x(0)=x(T), \quad x^{\prime}(0)=x^{\prime}(T)
\end{gathered}
$$

where $s$ is a real parameter, $g:[0, T] \times \mathbb{R} \rightarrow \mathbb{R}$ is a Carathéodory function and $k \in \mathbb{R} \backslash\{0\}$. We give conditions for periodic BVP (4.1)-(4.2) to have at least one solution by using the existence result obtained in Section 2 and Section 3.

Under the nonincreasing property of $g(t, x)$ with respect to the second variable, by applying Theorem 2.2 , one has

Theorem 4.1. Assume that there exist two constants $R_{1}>0, s_{1} \in \mathbb{R}$ and $g_{0} \in$ $L^{1}(I)$, such that

$$
g(t, x) \geq g_{0}(t)
$$

and

$$
g(t, 0) \leq s_{1} \leq \frac{1}{T} \int_{0}^{T} g_{0}(t) d t
$$


for a.e. $t \in I$ and all $x \geq R_{1}$. Then there exists an $s_{0} \leq s_{1}$ (with the possibility $\left.s_{0}=-\infty\right)$ such that

1) for $s<s_{0}$, (4.1)-(4.2) has no solution;

2) for $s \in\left(s_{0}, s_{1}\right]$, (4.1)-(4.2) has at least one solution.

Proof. We first prove the existence of a solution of problem (4.1)-(4.2) for $s=s_{1}$. Consider a periodic boundary value problem

$$
\begin{gathered}
\alpha^{\prime \prime}+k \alpha^{\prime}+g_{0}(t)-\frac{1}{T} \int_{0}^{T} g_{0}(t) d t=0, \\
\alpha(0)=\alpha(T), \quad \alpha^{\prime}(0)=\alpha^{\prime}(T) .
\end{gathered}
$$

In order to show that Eq. (4.5)-(4.6) has a solution $\alpha \in W^{2,1}(0, T)$, we consider the homotopy

$$
\alpha^{\prime \prime}+k \lambda \alpha^{\prime}+g_{0}(t)-\frac{1}{T} \int_{0}^{T} g_{0}(t) d t=0
$$

with (4.6) and $\lambda \in[0,1]$.

Multiplying (4.7) by $\alpha$ and integrating from 0 to $T$, we get

$$
\begin{aligned}
\left|\alpha^{\prime}\right|_{L^{2}}^{2} & =\left|\int_{0}^{T} \alpha\left(g_{0}(t)-\frac{1}{T} \int_{0}^{T} g_{0}(t)\right) d t\right| \leq|\alpha|_{\infty}\left|g_{0}(t)-\frac{1}{T} \int_{0}^{T} g_{0}(t) d t\right|_{L^{1}} \\
& \leq \sqrt{T}\left|\alpha^{\prime}\right|_{L^{2}}\left|g_{0}(t)-\frac{1}{T} \int_{0}^{T} g_{0}(t) d t\right|_{L^{1}}
\end{aligned}
$$

and therefore,

$$
\left|\alpha^{\prime}\right|_{L^{2}} \leq \sqrt{T}\left|g_{0}(t)-\frac{1}{T} \int_{0}^{T} g_{0}(t) d t\right|_{L^{1}}
$$

Hence,

$$
|\alpha|_{\infty} \leq T\left|g_{0}(t)-\frac{1}{T} \int_{0}^{T} g_{0}(t) d t\right|_{L^{1}},
$$

which shows that all the solutions of (4.7)-(4.6) will be bounded in $L^{1}(I)$.

By Mawhin's degree theorem [1], it follows that Eq. (4.5)-(4.6) has a solution $\alpha=\alpha(t)$ which satisfies $\int_{0}^{T} \alpha(t) d t=0$ and (4.9).

Now, let

$$
\bar{\alpha}_{0}=\bar{\alpha}-T\left|g_{0}(t)-\frac{1}{T} \int_{0}^{T} g_{0}(t) d t\right|_{L^{1}}-R_{1} .
$$

Then, by (4.3) and (4.4),

$$
g\left(t, \bar{\alpha}_{0}(t)\right) \geq g_{0}(t) \geq g_{0}(t)-\frac{1}{T} \int_{0}^{T} g_{0}(t) d t+s_{1}
$$

corresponds to a reversedly upper solution $\beta(t) \equiv 0$, according to (4.4).

Thus, Theorem 2.2 provides the existence of a solution $x_{1}(t)$ of problem (4.1)(4.2) for $s=s_{1}$, and

$$
\alpha_{0}(t) \leq x_{1}(t) \leq 0
$$

for all $t \in I$.

Next, we show that if problem (4.1)-(4.2) has a solution $x(t)$ for some $s<s_{1}$, then it has a solution for each $\widetilde{s} \in\left[s, s_{1}\right]$. 
Obviously, $x(t)$ is a reversedly upper solution of problem (4.1)-(4.2) (with $\widetilde{s}$ in place of $s$ ), which corresponds to a reversedly lower solution $\bar{\alpha}_{0}(t)$ of $(4.1)-(4.2)$ for $\widetilde{s}$, if we choose $R_{1}$ large enough to satify both $x(t)>-R_{1}$ and (4.3). $\widetilde{s}$.

Again, Theorem 2.2 yields the existence of a solution of problem (4.1)-(4.2) for

Finally, let us take $s_{0}=\sup \{s \in \mathbb{R} \mid(4.1)-(4.2)$ has at least one solution $\}$. If (4.1)-(4.2) has a solution for all $s \leq s_{1}, s_{0}$ will be taken as $-\infty$. From the discussion above, we have $s_{0} \leq s_{1}$ and that (4.1)-(4.2) has at least one solution for $s \in\left(s_{0}, s_{1}\right]$. Theorem 4.1 is proved.

\section{ACKNOWLEDGEMENTS}

The author of this paper wishes to express his gratitude to the referee for useful comments and suggestions. He also thanks Professor Shuxiang Yu for his advice and suggestions.

\section{REFERENCES}

1. Gains, R.E., and Mawhin, J., Concidence Degree and Nonlinear Differential Equations. Lecture Notes in Mathematics 56. Springer, Berlin, 1977. MR 58:30551

2. Habets, P., and Metzen, G., Existence of periodic solutions of Duffing equations, J. of Diff. Eqns. Vol 78(1989) pp. 1-32. MR 90c:34040

3. Habets, P., and Sanchez, L., Periodic solutions of some Liénard equations with singularities. Proceedings of the American Mathematical Society, Vol 109(1990) pp. 1035-1044. MR 90k:34049

4. Hartman,P., Ordinary Differential Equations. Second Edition. Birkhäuser, Boston, 1982. MR 83e:34002

5. Mawhin, J., Points fixes, ponits critiques et probléme aux limites. Semin. Math. Sup. no. 92. Press Univ. de Montreál, 1985.

6. Nkashama, M.N., A generalized upper and lower solutions method and multiplicity results for nonlinear first-order ordinary differential equations. J. of Math. Anal. Applic. Vol 140(1989) pp. 381-395. MR 90e:34006

7. Nieto, J.J.,et al A generalization of the monotone iterative technique for nonlinear second order oeriodic boundary value problem. J. of Math. Anal. Applic. Vol 151(1990) pp. 181-189. MR 91h:34025

Institute of Mathematics, Academia Sinica, Beijing 100080, People's Republic of China

Department of Mathematics \& Computer Science, Rutgers University, Newark, New JERSEY 07102

E-mail address: chengwen@pegasus.rutgers.edu 